\title{
MODELING A WELL STIMULATION PROCESS USING THE MEOR TECHNIQUE
}

\author{
Nmegbu C.G.J. ${ }^{1}$, Pepple D.D ${ }^{2}$ \\ ${ }^{1,2}$ Department of Petroleum Engineering, Rivers State University of Science and Technology, Rivers State, Nigeria
}

\begin{abstract}
Microbial enhanced oil recovery remains the most environmental friendly, cost effective recovery technique in oil production, particularly for wellbore stimulation. This research investigates the effects of microbial growth rate, microbial and nutrient concentrations for well stimulation purposes. A representative model incorporating microbial concentration, its growth rate and skin factor is developed, validated and discussed. An explicit formulation which poses a solution to the equation for the model is used to describe the reservoir pressure responses. It is observed through plots of reservoir pressure against reference distances that flow and production rates improved as a result of an improved BHP when the microbial parameters were incorporated to the fluid transport equation at same injection rates and same reservoir parameters. The trend followed by the pressure profile plots correlates with that expected of a well stimulation pressure profile.
\end{abstract}

Keywords: Well Stimulation, MEOR, Permeability, MEOR stimulation

\section{INTRODUCTION}

The current need for maximizing oil recovery from the reservoir has prompted the evaluation of improved oil recovery methods and various EOR techniques. Microbial EOR is an of aspect biotechnology, utilizing the potentials of microbes to significantly influence oil flow and its recovery. However, a sound and reliable engineering technique in optimizing microbial formulations are required to maximize these potentials.

The use of microbes for hydrocarbon recovery has been credible, and loss of crude during the process can be considered insignificant compared to the amount of increased recovery. Pressure, salinity, pore structure and mainly temperature often limit the functionality of microbes during any MEOR application.

The patented process described by ZoBell showed that bacterial products such as gases, acids, solvents, surfactants and cell biomass released oil from sand packed columns in a laboratory test [1]. Subsequent studies have shown that [2];

1. Viable bacteria and various nutrients required for growth can be transported through cores.

2. Insitu growth of bacteria results in significant reduction in formation permeability.

3. Permeability reduction is selective for high permeability cores and improves sweep even under conditions where cross flow of fluids between regions occur.

Taylor et al conducted a theoretical and experimental investigation to effectively quantify reduction in permeability as a result of enhanced microbial growth in a porous media [3]. They observed that enhanced biological activities in sand column reactors can significantly reduce permeability. An analytical relationship was then established between the biofilm thickness and resulting permeability reduction.

A one-dimensional, two-phase, compositional numerical simulator to model the transport and growth of bacteria and oil recovery in MEOR process was developed by Sarkar et al. [4]. In their model, permeability reduction was modeled using the effective medium theory an implicit-pressure, explicitconcentration algorithm was used to solve pressure and mass concentration equations.

Islam presented a mathematical formulation to describe and explain microbial transport in a multiphase multi directional flow through a porous media [5]. In his formulation, physical dispersion terms were neglected in the component transport equation, since metabolic product actions were not included in the model, considerations which relate biomass to metabolic and their activities were defined.

Nielson et al used a correlation between IFT and surfactant concentration. Usually, a reduction in IFT causes a decrease in residual oil saturations, therefore affecting the permeability curve end points [2]. They investigated the following methods $[2,6,7]$

1. Capillary number and normalized residual oil saturation correlations.

2. Coats interpolated between $K_{r}$ and the interpolation of factors of core types relative to permeability curves. 
They recommended the latter, in which more parameters can be estimated in order to obtain a better fit with experimental data.

Knapp et al also developed a 1 -Dimensional mathematical model to effectively describe the microbial plugging process [8]. The impact of cellular growth and microbial retention on temporal reduction in permeability of porous media were the main objectives investigated by this model. They assumed the development of stationary phase is solely due to the biomass retention therefore convective transport is the dominant mechanism for microbial mobilization. Their governing equation included a convection dispersion equation for bacteria and nutrient transport, and a mass conversion equation for stationary phase development.

Zhang et al presented a three-phase, multiple species, onedimensional mathematical model to simulate biomass growth, bioproduct formation, and substrate consumption during insitu microbial growth, and to predict permeability reduction as a result of in-situ growth and metabolism in porous media [9]. All the model parameters considered by respective authors are ideal for successful MEOR implementation. They are considered the most relevant as a result of multiple microbial oil recovery studies and mainly include microbial transport, microbial concentration, Interfacial Tension (IFT) reduction parameters, microbial kinetics, mobility control, viscosity reduction etc. This study basically aims at examining microbial formulations that can be applied as well stimulation alternatives and permeability alteration agents.

\section{MATERIALS AND METHOD}

The fundamental theories of Fluid flow and Monod growth kinetics would serve as a basis for modeling well stimulation processes by MEOR application as a result of permeability alteration caused by metabolite production by the choice microbe (clostridium $\mathrm{sp}$ ).

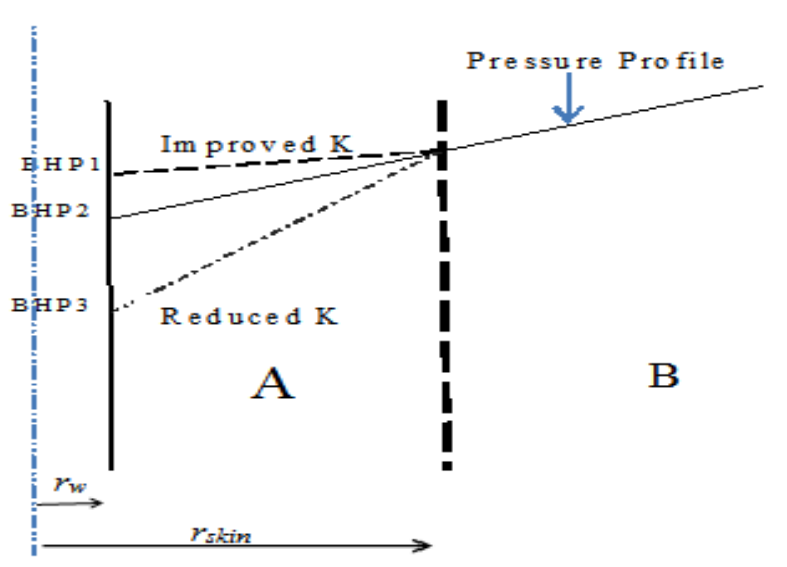

Region $\mathrm{A}$ is the damaged region, while region $\mathrm{B}$ is the undamaged region.

\subsection{Choice of Microbe}

Having the ability to effectively withstand reservoir with the most challenging conditions, particularly temperature and salinity, Clostridium sporonges proves the best stimulation microbe, prior to its metabolite production (butanol$\mathrm{CH}_{3}\left(\mathrm{CH}_{2}\right)_{3} \mathrm{OH}$ and acetones $\left(2 \mathrm{CH}_{3} \mathrm{COOH}\right)$ that alters the absolute permeability of reservoir rock after reaction to produce calcium acetate, carbon dioxide and water.

Being a thermophile, with a temperature tolerance range of about $50-70^{\circ} \mathrm{C}\left(122-158^{\circ} \mathrm{F}\right)$, this microbe can thrive in relatively high reservoir temperature condition, averaging about $60^{\circ} \mathrm{C}\left(140^{\circ} \mathrm{F}\right)$

Pores must be twice the diameter of the microbe for effective transportation to occur. Ideally, clostridium $s p$ records about $4.0 \mu \mathrm{m}$ length and $0.6 \mu \mathrm{m}$ thick. This proves convenient enough to be transported in a carbonate pore throat averaging $1.16 \mu \mathrm{m}$ minimum. An optimum $\mathrm{pH}$ for microbial existence and transport in the porous media lies between $4.0-9.0$, and clostridium sp lies between this limit (4.5 - 4.7).

\section{MATHEMATICAL MODELING}

\subsection{Microbial Growth Rate}

The growth rate expression applied for bacteria are often the Monod expression based on the Michelis-Menten enzyme kinetics [2, 5, 11]. The Monod expression with one limiting substrate is widely used, but it is empirical in the context of microbial growth.

The Monod growth rate for one limiting substrate without any inhibition will be used in this work:

$$
\dot{G}=G_{\max } \frac{C_{n}}{k_{s}+C_{n}}
$$

Where

$\mathrm{G}_{\max }$ is the maximum growth rate obtained in excess nutrient $\left(\mathrm{hr}^{-1}\right)$

$\mathrm{k}_{\mathrm{s}}$ is the substrate concentration to half $\mathrm{G}_{\max }(\mathrm{mg} / \mathrm{l})$ $\mathrm{C}_{\mathrm{n}}$ is the nutrient concentration $(\mathrm{mg} / \mathrm{l})$

\subsection{Fluid Transport Equation}

The most general form of a single phase fluid flow equation in a porous media is presented below in equation (2), making no assumptions regarding fluid type or pressure dependency on rock and fluid properties [12];

Fig -1: Effects of well bore vicinity damage on the pressure profile and BHP levels [10] 


$$
\begin{gathered}
\frac{\partial}{\partial x}\left(U_{x}\right) \frac{1}{B} \Delta x+\frac{\partial}{\partial y}\left(U_{y}\right) \frac{1}{B} \Delta y+\frac{\partial}{\partial z}\left(U_{z}\right) \frac{1}{B} \Delta z+q= \\
\frac{v_{b} \phi S_{i} C_{t}}{\alpha_{c} B_{o}} \frac{\partial P}{\partial t} \frac{\partial}{\partial x}\left(U_{x}\right) \frac{1}{B} \Delta x+\frac{\partial}{\partial y}\left(U_{y}\right) \frac{1}{B} \Delta y+\frac{\partial}{\partial z}\left(U_{z}\right) \frac{1}{B} \Delta z+q= \\
\frac{v_{b} \phi S_{i} C_{t}}{\alpha_{c} B_{o}} \frac{\partial P}{\partial t}
\end{gathered}
$$

\subsection{Assumptions for Model Development}

1. Fluid flow is one-dimensional single phase, and takes place in a uniform porous medium.

2. Metabolite production mostly bioacids [2]

3. Isothermal system as reservoir fluctuations in temperature is regarded minimal [4]

4. A change in temperature will alter the individual values of $C_{t}, \mu_{o}, B$ and $P$

5. No break in injection rates of nutrient and bacteria during the process

6. Microbial decay not considered.

7. No indigenous bacteria present.

8. Flow in the reservoir is in the direction of the wellbore.

9. Chemotaxis (movement of microbes towards an increasing concentration of substrate) not considered.

10. No substrate and metabolite adsorption on the pore walls, so Langmuir equilibrium isotherm not considered.

11. Flow is laminar (reservoir contains only oil).

12. Unsteady state flow conditions.

13. Other factors affecting growth rates such as salinity and $\mathrm{pH}$ remain constant.

With these assumptions imposed on equation (2) the flow of fluid in the reservoir was

Representing a 1-dimensional, single phase flow system as:

$$
\frac{\partial}{\partial x}\left(U_{x}\right) \frac{1}{B} \Delta x+q=\frac{v_{b} \phi C_{t}}{\alpha_{c} B_{o}} \frac{\partial P}{\partial t}
$$

From Darcy’s law for a 1-dimensional flow system:

$$
U_{x}=-\beta_{c} \frac{A_{x} k_{x}}{\mu_{o}} \frac{d P}{d x}
$$

Substituting equation (4) into (3), we have:

$$
\frac{\partial}{\partial x}\left(-\beta_{c} \frac{A_{x} k_{x}}{\mu_{o}} \frac{d P}{d x}\right) \frac{1}{B} \Delta x+q=\frac{v_{b} \phi C_{t}}{\alpha_{c} B_{o}} \frac{\partial P}{\partial t}
$$

Accounting for microbial concentration[2]:

$$
\frac{\partial}{\partial x}\left(-\beta_{c} \frac{A_{x} k_{x} C_{b}}{\mu_{o}} \frac{d P}{d x}\right) \frac{1}{B} \Delta x+q C_{b}=\frac{v_{b} \phi C_{t} C_{b}}{\alpha_{c} B_{o}} \frac{\partial P}{\partial t}
$$

Equation (6) is known as the component transport equation for microbes.

For slightly compressible fluids such as oil,
Formation volume factor, $\mathrm{B}=\frac{B_{o}}{1+c\left[P-P_{o}\right]}$

For initial boundary conditions,

$\mathrm{P}=\mathrm{P}_{\mathrm{o}}$, therefore $\mathrm{B}=\mathrm{B}_{\mathrm{o}}$.

Neglecting the negative sign on the LHS of equation (6), the equation is reduced to:

$$
\frac{\partial}{\partial x}\left(\beta_{c} \frac{A_{x} k_{x} C_{b}}{\mu_{o} B} \frac{d P}{d x}\right) \Delta x+q C_{b}=\frac{v_{b} \phi C_{t} C_{b}}{\alpha_{c} B} \frac{\partial P}{\partial t}
$$

Incorporating the Monod equation to account for microbial growth rate, we have;

$$
\frac{\partial}{\partial x}\left(\beta_{c} \frac{A_{x} k_{x} C_{b}}{\mu_{o} B} \frac{d P}{d x}\right) \Delta x+q C_{b}+\dot{G}=\frac{v_{b} \phi C_{t} C_{b}}{\alpha_{c} B} \frac{\partial P}{\partial t}
$$

For stimulation, skin factor must be considered (showing the relativity of permeability and radii of investigation ), and is represented as thus [10];

$$
S=\left[\frac{k}{k_{\text {skin }}}-1\right] \ln \left(\frac{r_{\text {skin }}}{r_{w}}\right)
$$

Incorporating skin factor into equation (8), we have

$$
\frac{\partial}{\partial x}\left(\beta_{c} \frac{A_{x} k_{x} C_{b}}{\mu_{o} B} \frac{d P}{d x}\right) \Delta x+q C_{b}+\dot{G}=\frac{v_{b} \phi C_{t} C_{b} S}{\alpha_{c} B} \frac{\partial P}{\partial t}
$$

Equation (10) can be used to predict pressure in the reservoir after microbial injection.

\section{SOLUTION TO MATHEMATICAL FORMULATION}

Rewriting equation (10) as a second order derivative, we have:

$$
\frac{\partial^{2} P}{\partial x^{2}}\left(\beta_{c} \frac{A_{x} k_{x} C_{b}}{\mu_{o} B}\right) \Delta x+q C_{b}+\dot{G}=\frac{v_{b} \phi C_{t} C_{b} S}{\alpha_{c} B} \frac{\partial P}{\partial t}
$$

Applying central difference approximation in space $(x)$ and forward difference approximation in time $(t)$, we have [12];

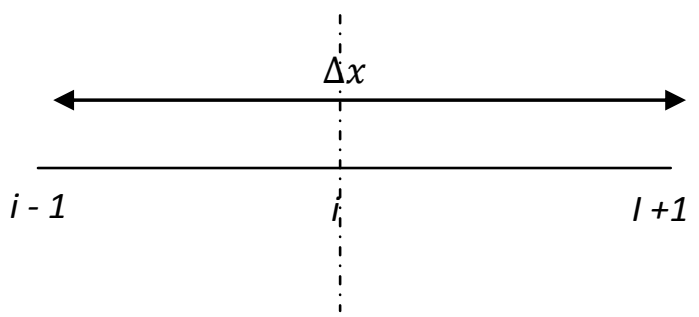

Fig -2: Discrete points representation (grid positions)

$$
\frac{\partial^{2} P}{\partial x^{2}}=\frac{P_{i+1}^{n}-2 P_{i}^{n}+P_{i-1}^{n}}{\Delta x^{2}} \quad \text { and }
$$




$$
\frac{\partial P}{\partial t}=\frac{P_{i}^{n+1}-P_{i}^{n}}{\Delta t}
$$

Where ' $i$ ' is position and ' $n$ ' is the time step. Applying the approximations to Equation (11) gives:

$$
\begin{aligned}
& \frac{P_{i+1}^{n}-2 P_{i}^{n}+P_{i-1}^{n}}{\Delta x^{2}}\left(\beta_{c} \frac{A_{x} k_{x} C_{b}}{\mu_{o} B}\right) \Delta x+\left(q C_{b}+\dot{G}\right)= \\
& \left(\frac{v_{b} \phi C_{t} C_{b} S}{\alpha_{c} B}\right) \frac{P_{i}^{n+1}-P_{i}^{n}}{\Delta t}
\end{aligned}
$$

Rearranging equation (12) we have:

$$
\begin{gathered}
\left(\beta_{c} \frac{A_{x} k_{x} C_{b}}{\mu_{o} B}\right) \Delta x \frac{P_{i+1}^{n}}{\Delta x^{2}}-2\left(\beta_{c} \frac{A_{x} k_{x} C_{b}}{\mu_{o} B}\right) \Delta x \frac{P_{i}^{n}}{\Delta x^{2}}+ \\
\left(\beta_{c} \frac{A_{x} k_{x} C_{b}}{\mu_{o} B}\right) \Delta x \frac{P_{i-1}^{n}}{\Delta x^{2}}+\left(q C_{b}+\dot{G}\right)= \\
\left(\frac{v_{b} \phi C_{t} C_{b} S}{\alpha_{c} B}\right) \frac{P_{i}^{n+1}-P_{i}^{n}}{\Delta t}
\end{gathered}
$$

The above can now be written as:

$$
\begin{gathered}
\left(\beta_{c} \frac{A_{x} k_{x} C_{b}}{\Delta x \mu_{o} B}\right) P_{i+1}^{n}-2\left(\beta_{c} \frac{A_{x} k_{x} C_{b}}{\Delta x \mu_{o} B}\right) P_{i}^{n}+ \\
\left(\beta_{c} \frac{A_{x} k_{x} C_{b}}{\Delta x \mu_{o} B}\right) P_{i-1}^{n}+\left(q C_{b}+\dot{G}\right)=\left(\frac{v_{b} \phi C_{t} C_{b} S}{\alpha_{c} B}\right) \frac{P_{i}^{n+1}-P_{i}^{n}}{\Delta t}
\end{gathered}
$$

Taking $\left(\beta_{c} \frac{A_{x} k_{x} C_{b}}{\Delta x \mu_{o} B}\right)$ to be $\mathrm{M}$, rewriting equation (14) gives:

$$
\begin{aligned}
& {\left[M P_{i+1}^{n}-2 M P_{i}^{n}+M P_{i-1}^{n}\right]+\left(q C_{b}+\dot{G}\right)=} \\
& \left(\frac{v_{b} \phi C_{t} C_{b} S}{\alpha_{c} B}\right) P_{i}^{n+1}-P_{i}^{n}
\end{aligned}
$$

For initial boundary conditions, all pressure values at any position ' $i$ ' at present time step ' $n$ ' are the same, so the values of $P_{i}^{n}$ and $P_{i-1}^{n}$ are all equal and known. The only unknown is the pressure value at position ' $\mathrm{i}$ ' at a new time step $n+1$.

In order to make $P_{i}^{n+1}$ the subject, we first multiply through equation (15) by the inverse of $\frac{v_{b} \phi C_{t} C_{b} S}{\alpha_{c} B \Delta t}$, we have;

$$
\begin{gathered}
\left(\frac{\alpha_{c} B \Delta t}{v_{b} \phi C_{t} C_{b} S}\right)\left[M P_{i+1}^{n}-2 M P_{i}^{n}+M P_{i-1}^{n}\right]+ \\
\left(\frac{\alpha_{c} B \Delta t}{v_{b} \phi C_{t} C_{b} S}\right)\left(q C_{b}+\dot{G}\right)=P_{i}^{n+1}-P_{i}^{n}
\end{gathered}
$$

Let $\left(\frac{\alpha_{c} B \Delta t}{v_{b} \phi C_{t} C_{b} S}\right)=\mathrm{C}$, we can write;

$$
\begin{gathered}
C\left[M P_{i+1}^{n}-2 M P_{i}^{n}+M P_{i-1}^{n}\right]+C\left(q C_{b}+\dot{G}\right)=P_{i}^{n+1}- \\
P_{i}^{n}
\end{gathered}
$$

Equation (17) can now be written as thus;

$$
\begin{aligned}
& P_{i}^{n+1}=P_{i}^{n}+C\left[M P_{i+1}^{n}-2 M P_{i}^{n}+M P_{i-1}^{n}\right]+ \\
& C\left(q C_{b}+\dot{G}\right)
\end{aligned}
$$

\section{MODEL VALIDATION}

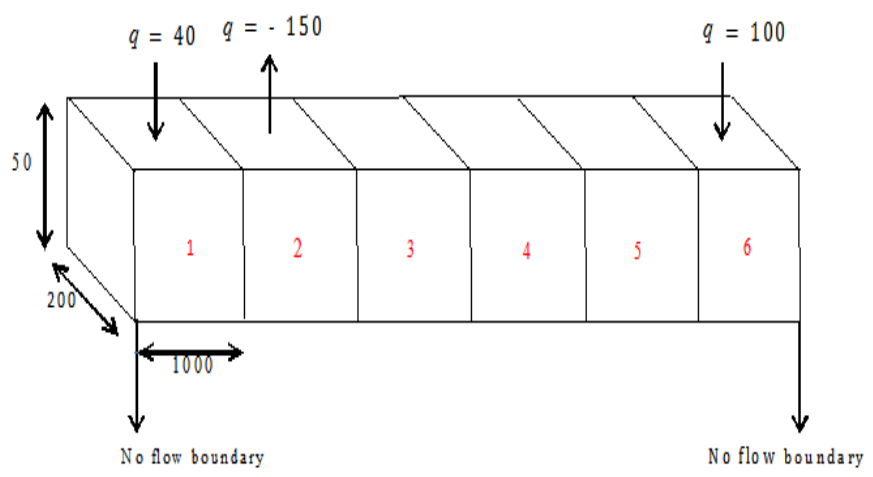

Fig -3: Discretization of reservoir showing dimensions,production and injection points

Table -1: Field parameters

\begin{tabular}{|l|l|}
\hline Parameters & Values \\
\hline Depleted reservoir pressure & $1500 \mathrm{psi}$ \\
\hline $\begin{array}{l}\text { Permeability of damaged } \\
\text { zone }\left(K_{\text {skin }}\right)\end{array}$ & $30 \mathrm{ft}$ \\
\hline Total compressibility, $C_{t}$ & $10^{*} 10^{-6} \mathrm{psi}^{-1}$ \\
\hline Transmissibility coefficient & 1.127 \\
\hline Formation porosity & $20 \%$ \\
\hline Wellbore radius, $r_{w}$ & $0.25 \mathrm{ft}$ \\
\hline Damaged radius, $r_{\text {skin }}$ & $2 \mathrm{ft}$ \\
\hline Formation permeability, $\mathrm{k}$ & $160 \mathrm{md}$ \\
\hline Volume conversion factor, $\alpha_{c}$ & 5.615 \\
\hline$\Delta x$ & $1000 \mathrm{ft}$ \\
\hline$\Delta y$ & $50 \mathrm{ft}$ \\
\hline$\Delta z$ & $200 \mathrm{ft}$ \\
\hline $\begin{array}{l}\text { Oil formation volume factor, } \\
B_{o}\end{array}$ & $1.00 \mathrm{rb} / \mathrm{stb}$ \\
\hline Oil viscosity, $\mu_{o}$ & $10 \mathrm{cp}$ \\
\hline $\begin{array}{l}\text { Permeability of damaged zone, } \\
K_{\text {skin }}\end{array}$ & $30 \mathrm{md}$ \\
\hline$\Delta t$ & $30 \mathrm{days}$ \\
\hline
\end{tabular}


Table -2: Nutrient and microbial parameters [11]

\begin{tabular}{|l|l|}
\hline Parameters & Values \\
\hline $\begin{array}{l}\text { Max microbial growth } \\
\text { rate, } G_{\max }\end{array}$ & $0.343 \mathrm{hr}^{-1}$ \\
\hline $\begin{array}{l}\text { The substrate concentration at } \\
\text { half } \mathrm{G}_{\max }, \mathrm{k}_{\mathrm{s}}\end{array}$ & $12.8(\mathrm{mg} / \mathrm{l})$ \\
\hline Nutrient concentration, $C_{n}$ & $45 \mathrm{mg} / \mathrm{l}$ \\
\hline Microbial concentration, $C_{b}$ & $\begin{array}{l}3.5 \times 10^{-4} \frac{\text { cells }}{\mathrm{ml}}= \\
10 \text { cells } / \mathrm{ft}^{3}\end{array}$ \\
\hline
\end{tabular}

\subsection{Calculation of Constants}

Skin factor, $\mathrm{S}=\left[\frac{K}{K_{\text {skin }}}-1\right] \ln \left[\frac{r_{\text {skin }}}{r_{w}}\right]$

Microbial growth rate, $\dot{G}=G_{\max } \frac{C_{n}}{k_{s}+C_{n}}$

Constants, $C=\frac{\alpha_{c} B_{o} \Delta t}{v_{b} \phi C_{t} C_{b} S}$

Transmissibility term, $M=\frac{\beta_{C} A_{x}\left(\frac{K_{\text {avg }}}{1000}\right)_{x} C_{b}}{\mu B_{o} \Delta x}$

We have;

$\mathrm{S}=9.01$

$\dot{G}=6.4$ day $^{-1}$

$\mathrm{C}=0.093$

$\mathrm{M}=1.071$

\subsection{Calculation of Pressure Responses at Different}

\section{Grid Blocks}

For time step $1, \Delta t=30$ days

Setting initial boundary conditions, $\mathrm{P}_{\mathrm{i}}=1500 \mathrm{psi}$

Recalling,

$$
P_{i}^{n+1}=P_{i}^{n}+C\left[M P_{i+1}^{n}-2 M P_{i}^{n}+M P_{i-1}^{n}\right]+C\left(q C_{b}+\dot{G}\right)
$$

For grid block 1, i=1

$$
\begin{array}{r}
P_{1}^{n+1}=1500+0.093[1.071(1500)-(2 \times 1.071)(1500) \\
+1.071(1500)]+0.093(40(10)+6.4)
\end{array}
$$

$P_{1}^{n+1}=1537.79 p s i$

For grid block 2, i=2

$$
\begin{aligned}
P_{2}^{n+1}=1500+ & 0.093[1.071(1500)-(2 \times 1.071)(1500) \\
& +1.071(1500)] \\
& +0.093(-150(10)+6.4)
\end{aligned}
$$

$P_{2}^{n+1}=1360.39 p s i$
For grid block 3, i=3

$$
P_{3}^{n+1}=1500+0.093[1.071(1500)-(2 \times 1.071)(1500)
$$$$
+1.071(1500)]+0.093(0(10)+6.4)
$$

$P_{3}^{n+1}=1500.60 p s i$

For grid block 4, i=4

$$
P_{4}^{n+1}=1500+0.093[1.071(1500)-(2 \times 1.071)(1500)
$$$$
+1.071(1500)]+0.093(0(10)+6.4)
$$

$P_{4}^{n+1}=1500.60 p s i$

For grid block 5, i=5

$P_{5}^{n+1}=1500+0.093[1.071(1500)-(2 \times 1.071)(1500)$ $+1.071(1500)]+0.093(0(10)+6.4)$

$P_{5}^{n+1}=1500.60 p s i$

For grid block 6, $\mathbf{i}=6$

$P_{6}^{n+1}=1500+0.093[1.071(1500)-(2 \times 1.071)(1500)$ $+1.071(1500)]+0.093(100(10)+6.4)$

$P_{6}^{n+1}=1594.07$

\section{RESULTS AND DISCUSSION}

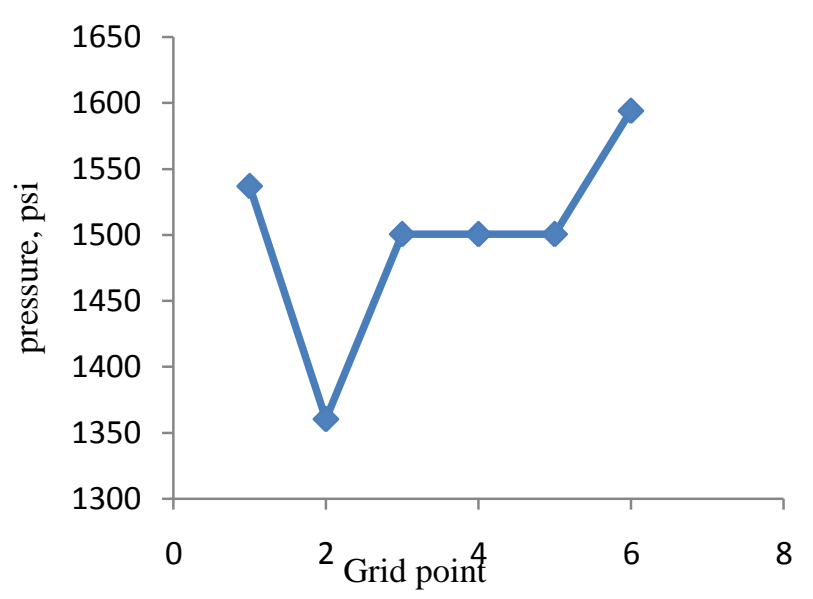

Fig -4: Pressure profile with MEOR model

The plot above shows a BHP of $1360.4 \mathrm{psi}$ at the production point at grid block 2

\subsection{Comparison with Fluid Flow Equation Excluding}

\section{Microbial Parameters and Skin Factor}

General fluid flow equation is given as:

$\frac{\partial}{\partial x}\left(\beta_{c} \frac{A_{x} k_{x}}{\mu_{o} B_{o}} \frac{d P}{d x}\right) \Delta x+q=\frac{v_{b} \phi C_{t}}{\alpha_{c} B_{o}} \frac{\partial P}{\partial t}$ 
Applying finite difference approximation to the above, we have:

$P_{i}^{n+1}=P_{i}^{n}+\left[\frac{\alpha_{c} B \Delta t}{v_{b} \phi C_{t}}\right] q+\left[\frac{\alpha_{c} B \Delta t}{v_{b} \phi C_{t}}\right]\left[M_{x_{i}+1 / 2} P_{i+1}^{n}-\right.$

$M x i+12+M x i-12 P i n+M x i-12 P i-1 n$

Where:

$\left[\frac{\alpha_{c} B \Delta t}{v_{b} \phi C_{t}}\right]$ is the injection or production rate factor,

$M_{x_{i} \pm 1 / 2}$ fluid transmissibility term, $M=\frac{\beta_{C} A_{x} K_{x}}{\mu B_{o} \Delta x}$

Solving for constants,

$\mathrm{C}=8.4225$

$M=0.107$

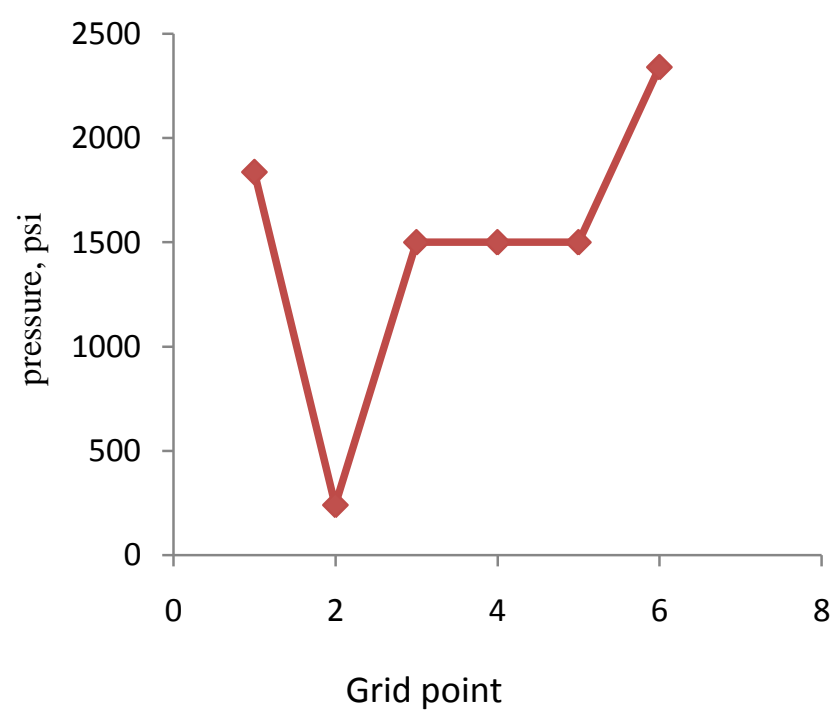

Fig -5: Pressure profile (with water) without MEOR

\begin{tabular}{|l|l|l|}
\hline Field result & Model prediction & Deviation \\
\hline $230 \mathrm{psi}$ & $240 \mathrm{psi}$ & $10 \mathrm{psi}$ \\
\hline
\end{tabular}

It is shown from figure 5 that a BHP of 240psi exists at the production point in grid block 2, with same injection and production rates, field parameters etc. used in the MEOR model. it is observed that there is a significant increase of pressure at the boundaries and injection points, but minimal pressure response at the production point. This implies that there is a resistance to fluid flow around the wellbore region, possibly skin effect which is responsible for the existence of a low pressure response at the BHP at the production point

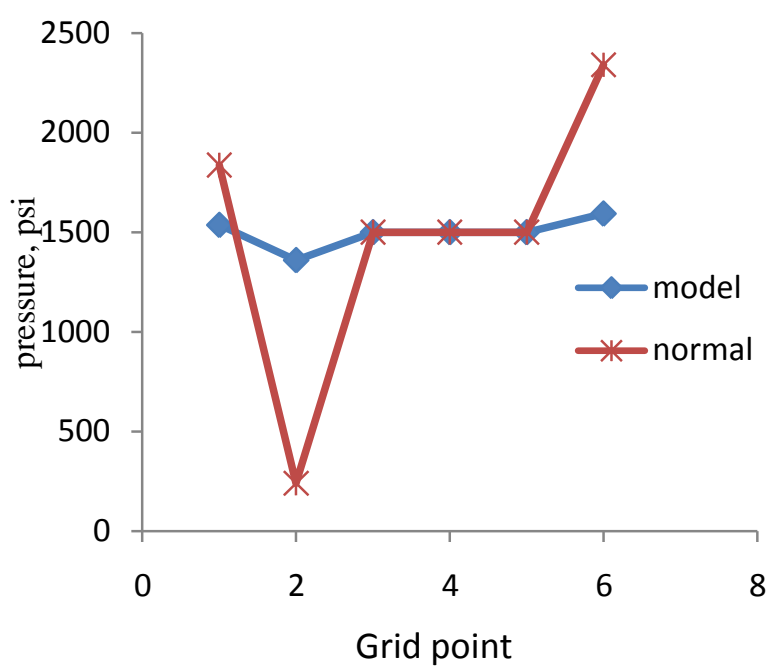

Fig -6: Pressure profiles comparison

\section{CONCLUSIONS}

The BHP levels in 1, 2 and 3 in fig 1.1 represents bottom hole pressure level at improved, ideal and reduced permeability respectively. The comparison of both models as shown in fig (1.6) correlates with the pressure profile for an improved wellbore vicinity as shown in fig (1.1). It is observed that the BHP records about 240psi before the Meor formulation. A bottom hole pressure of $1360.39 \mathrm{psi}$ is established after MEOR treatment, this pressure increase of about 1120.39psi implies that there is an improved oil flow towards the wellbore, prior to the improvement in permeability and damage reduction around the wellbore vicinity. It is recommended that an optimum microbial concentration must be investigated so as to ascertain a concentration limit to prevent microbial plugging or clogging.

\section{ACKNOWLEDGEMENTS}

The authors would love to appreciate the department of petroleum engineering RSUST for her relentless guidance and support in course of this research. The contributions of Dr. Jackson Akpa and Ohazuruike Lotanna, amongst others, are highly recognized.

\section{REFERENCES}

[1]. Lazar, I. and Constantinescu, P. (1997): Field trials results of MEOR. Microbes and Oil Recovery, 1: pp. 122-131.

[2]. Nielson, S.M., Shapiro, A.A., Michelsen, M.L. and Stenby, E.H. (2010). 1D simulations for microbial enhanced oil recovery with metabolite partitioning. Transp. Porous Media. 85: pp. 785-802.

[3]. Taylor SW, Milly PCD, Jaffe PR. (1990). Biofilm growth and the related changes in the physical-properties of a porousmedium. 2. Permeability. Water Resour Res 26:pp. 21612169. 
[4]. Sarkar, A., G. Georgiou, and M. Sharma (1994). Transport of bacteria in porous media: II. A model for convective transport and growth. Biotechnol Bioeng 44, pp. 499-508

[5]. Islam, M. (1990). Mathematical modeling of microbial enhanced oil recovery. SPE-20480 presented at ATCE, New Orleans, Louisiana, USA, 23-26 September

[6]. Coats, K. H. (1980). An equation of state compositional model. SPE J 20, pp. 363-376.

[7]. Al-Wahaibi, Y. M., C. A. Grattoni, and A. H. Muggeridge (2006). Drainage and imbibition relative permeabilities at near miscible conditions. J Petrol Sci Eng 53, pp. 239-253

[8]. Knapp RM, McInerney MJ, Coates JD, Chisholm JL, Menzie DE, Bhu-pathiraju VK. (1992). Design and Implementation of a Microbially Enhanced Oil Recovery Field Pilot, Payne County, Oklahoma. SPE 24818, Presented at the 1992 Annual Technical Conference and Exhibition, October 22-25, 1992, Dallas, Texas

[9]. Zhang, X., Knapp R., and McInerney M. (1992). A mathematical model for enhanced oil recovery process.

SPE-24202 presented at IOR Symposium, 22-24 April, Tulsa, Oklahoma, USA.

[10]. Ahmed, T (2010) Reservoir Engineering Handbook , 4th edition Massachusett, Gulf Professional Publishers, pp. 431434

[11]. Chang M.M., Chung, F., Bryant, R., Gao, H., and Burchfield T. (1991): Modeling and laboratory investigation of microbial transport phenomena in porous media. In: SPE 22845 presented at 66th Annual Technical Conference and exhibition of SPE in Dallas Texas.

[12]. Ertekin .J.T, Gregory E .R, Jamal.H.(2001) Basic Applied Reservoir Simulation .Henry Doherty memorial fund of AIME, Society Of Petroleum Engineers, Richardson, Texas. 